\title{
Restrições espaciais no controle motor de movimentos rápidos e precisos
}

\author{
Spatial constraints in the motor control of speed and accurate \\ movements
}

\author{
V.A. Okazaki, C.F. Pereira, F.A. Okazaki, J.B. Dascal
}

ARTIGO ORIGINAL | ORIGINAL ARTICLE

\begin{abstract}
RESUMO
Foi analisado o efeito de restrições espaciais (distância e tamanho do alvo) sobre as estratégias de controle motor em movimentos rápidos e precisos. Vinte homens (20-26 anos) realizaram a tarefa de Fitts simulada em computador. O software Discrete Aiming Task (v.1.0) forneceu a análise cinemática do cursor do mouse e a manipulação de índices de dificuldade ( 1 até 5 bits). O acréscimo na distância aumentou o $T M$, fase de aceleração, fase de desaceleração e velocidade, mas sem alterar a precisão. A manutenção na precisão foi explicada pela estratégia da manutenção na proporção entre as fases de aceleração e desaceleração. A redução do alvo proporcionou maiores $T M$ e fase de desaceleração e diminuição do maior valor de velocidade, que permitiram maior precisão mesmo com a manutenção na velocidade média. Diferentes estratégias de controle motor foram realizadas em função da restrição espacial manipulada pela distância e pelo tamanho do alvo.

Palavras-chave: restrição espacial, distância, tamanho do alvo, velocidade-precisão, controle motor
\end{abstract}

ABSTRACT

The effect of spatial constraints (distance and target width) was analyzed over the motor control strategies of speed and accurate movements. Twenty men (20-26 years old) performed the Fitts' task simulated in a computer. The Discrete Aiming Task (v.1.0) provided the kinematic analysis of the mouse cursor and the index of difficulties manipulation (1 to 5 bits). Distance increased provided great TM, acceleration phase, deceleration phase, and velocity, but without changing accuracy. Accuracy maintenance was explained by the maintenance of acceleration and deceleration phases' strategy. Target width decrease provided greater TM and deceleration phase and reduction of the peak velocity, which allowed better accuracy even when average velocity was held constant. Different control strategies were performed in function of the spatial constraint manipulated by the distance and target width. Keywords: spatial constraints, distance, target width, speed-accuracy, motor control

Submetido: 14.06.2012 | Aceite: 04.01.2013

Victor Hugo Alves Okazaki, Juliana Bayeux Dascal. Universidade Estadual de Londrina - Neurociências Motoras (NEMO), Brasil.

Carla Ferro Pereira. Universidade de São Paulo - Sistemas Motores Humanos (SMH), Brasil.

Fábio H. A. Okazaki. Instituto Compartilhar - Centro de Estudos do Movimento Humano, Brasil.

Endereço para correspondência: Prof. Dr. Victor Hugo Alves Okazaki, Departamento de Educação Física Universidade Estadual de Londrina, Campus Universitário, Rodovia Celso Garcia Cid Km 380, Caixa Postal: 6001, CEP 86051-990, Londrina - Paraná - Brasil.

E-mail:vhaokazaki@gmail.com 
Um dos principais objetivos em qualquer domínio da pesquisa é o estabelecimento de relações entre as variáveis dependentes e independentes (Zelaznik, 1993). No comportamento motor, a investigação da distância, do tempo e da precisão no movimento tem produzido a formulação de modelos capazes de explicar a relação destas variáveis (Elliot, Helsen, \& Chua, 2001). Woodworth (1899) foi o primeiro pesquisador a procurar estabelecer a relação entre estas variáveis e a analisar os processos de controle dos movimentos de precisão destinados a alvos espaciais. A partir de três experimentos (toques repetitivos em pequenos quadrados, toques repetitivos em três pequenos círculos dispostos formando um triângulo e traçar linhas retas em direção a áreas alvos perpendiculares) foi demonstrada menor precisão à medida que o tempo de movimento foi diminuído. Ou seja, o aumento na velocidade de movimento levaria à imprecisão de movimento.

Este paradigma da relação inversa velocidade-precisão teve maior visibilidade na comunidade científica após o trabalho de Paul Fitts (1954). Fitts (1954) levantou a hipótese de que a capacidade de transmissão da informação fixa do sistema motor possibilita a análise de uma informação métrica. Na qual, considerando uma tarefa de movimento, a informação seria transmitida através de um canal de comunicação estocástico de ruído que modela o comportamento do sistema motor humano. Fitts utilizou uma adaptação do teorema 17 de Shannon (1948), que expressa a capacidade de informação efetiva C (em bitts/segundo), de um canal com comprimento de banda B (em $\mathrm{Hz})$, resumida pela equação: $C=B \log 2[(S+$ $N) / N]$, no qual $N$ é a potência do ruído e $S$ é a potência do sinal. Adaptando estes conceitos da teoria da informação para o sistema motor humano, Fitts apontou que em taxa máxima de transmissão da informação, o sistema motor humano se comporta de acordo com uma relação logarítmica através da substituição de
$1 / M T$ por $B, 2 D$ por $S+N$, e $A$ por $N$ para obter sua equação de predição do comportamento: $M T=a+b \log 2(2 D / A)$, no qual $a$ e $b$ são constantes empíricas, $D$ é a distância de movimento e $A$ é o tamanho do alvo.

Fitts proporcionou suporte para sua hipótese através de três experimentos com tarefa de toques repetitivos com duas ponteiras (leve e pesada), tarefa de transferência de discos e tarefa de transferência de pinos. Através da manipulação na distância entre tamanho dos alvos e a distância entre eles (nestes três experimentos), foi demonstrado aumentou proporcional do TM com o acréscimo no ID, por meio do aumento na distância do movimento ou da diminuição do tamanho do alvo. Esta relação $T M \times I D$ obteve grande consistência de outros estudos que analisaram diferentes tarefas, tais como: discretas (Carlton, 1980; Guiard, 1997; Jax, Rosenbaum, \& Vaughan, 2007), cíclicas (Crossman \& Goodeve, 1963/1983; Fitts, 1954; Kvalseth, 1975), com mouse (Boritz, Booth, \& Cowan, 1991; Jonhsgard, 1994; Okazaki, Okazaki, Lima, Caminha, \& Teixeira, 2008), com joystick (Epps, 1986; Jagacinski, Repperger, Moran, Ward, \& Glass, 1980), de rotação de punho (Meyer, Smith, \& Wright, 1982; Wright \& Meyer, 1983), de extensão de braço (Kerr \& Langolf, 1977), flexão de braço (Corcos, Gottlieb, \& Agarwal, 1988), etc.. Tal sustentação legitimou esta formulação matemática para um dos fenômenos mais consistentes em comportamento motor, o que induziu o paradigma ao seu atual status de 'Lei de Fitts' (Bootsma, Fernandez, \& Mottet, 2004; Sparrow \& Sparrow, 1991).

Apesar da grande consistência nas predições realizadas pela lei de Fitts, têm sido apontadas algumas limitações em relação à contribuição das restrições espaciais utilizadas na determinação do ID (Meyer, Abrams, Kornblum, Wright, \& Smith, 1988; Sheridan, 1979; Welford, Norris, \& Shock, 1969). Por exemplo, tem sido demonstrado que há um aumento na desproporcionalidade causada no ID através de 
reduções no tamanho do alvo, quando comparado aos aumentos similares na distância entre os alvos (Buck, 1986; Jagacinski \& Mok, 1985; Keele, 1973; Meyer et al., 1988; Welford et al., 1969). Este efeito particular das restrições espaciais também tem sido verificado nas análises da taxa do erro (tentativas em que os alvos não conseguem ser atingidos), a qual aumenta com a diminuição no tamanho do alvo independentemente da distância entre os alvos (Card, English, \& Burr, 1978; Wade, Newell, \& Wallace, 1978). Por conseguinte, as restrições espaciais da distância de movimento e do tamanho do alvo parecem ser reguladas por estratégias de controle particulares. Nas quais, a manipulação na distância de movimento estaria mais relacionada aos aspectos do controle da velocidade/aceleração do sistema efetor. Desta forma, o aumento na restrição espacial por meio de maiores distâncias proporcionará grandes velocidades (média e máxima), resultando em menor precisão (maior variabilidade do alvo efetivo). Ao passo que, a manipulação no tamanho do alvo envolveria os aspectos relacionados à acurácia do movimento. Assim, o aumento na restrição espacial através da diminuição no tamanho do alvo levará às menores velocidades (média e máxima), resultando em maior precisão (menor variabilidade do alvo efetivo). Todavia, apesar das características particulares das restrições espaciais verificadas em estudos posteriores, a comparação entre o efeito das restrições da distância de movimento e do tamanho do alvo não tem sido foco de estudo no paradigma da relação inversa velocidade-precisão.

O presente estudo analisou o efeito das restrições espaciais da distância e do tamanho do alvo sobre as estratégias de controle motor em movimentos rápidos e precisos. Espera-se contribuir para o entendimento das diferentes estratégias de controle motor utilizadas na regulação de movimentos rápidos e precisos submetidos aos diferentes tipos restrições espaciais (distância e alvo).

\section{MÉTODO}

\section{Amostra}

Participaram do estudo 20 universitários homens com idade entre 20 e 26 anos. Todos os participantes assinaram um termo de consentimento livre e esclarecido de participação. Os procedimentos do estudo foram aprovados pelo Comitê de Ética em Pesquisa da universidade local.

\section{Instrumentos}

Foi utilizada a tarefa de toque discreto realizada em um ambiente virtual através de um software especialmente desenvolvido para este estudo (Discrete Aiming Task v.1.0; Okazaki, 2006) simulando a tarefa Fitts (cf. Fitts, 1954). A tarefa consistiu em clicar em duas placas paralelas, nas quais eram manipulados três tamanhos de alvo ( $A=1,0.5$ e 2.5 polegadas) e três distâncias de movimento $(D=2,4$ e 8 polegadas) para fornecer índices de dificuldade de 3 até $5([I D=\log 2(2 D / A)])$. O software forneceu a análise cinemática do cursor do mouse (deslocamento linear em função do tempo). Foi utilizado um mouse óptico (marca Knex) com o nível de sensibilidade configurado para 50\%. Como superfície para deslizamento do mouse foi utilizado um "mouse pad" medindo $19 \mathrm{~cm} \times 23 \mathrm{~cm}$ de cor preta. A figura 1 apresenta uma representação esquemática do software utilizado para a realização da tarefa experimental.

\section{Procedimentos}

Inicialmente os participantes foram informados dos procedimentos experimentais e assinaram um termo de consentimento de participação livre e esclarecido. Para confirmar a preferência manual para o lado direito (sujeitos destros) foi aplicado o Inventário de Dominância Lateral de Edimburgo (Oldfield, 1971), constituído por 10 questões sobre preferência lateral na realização de 10 tarefas motoras do cotidiano. Em seguida, foi solicitado aos sujeitos para sentarem em uma posição confor- 


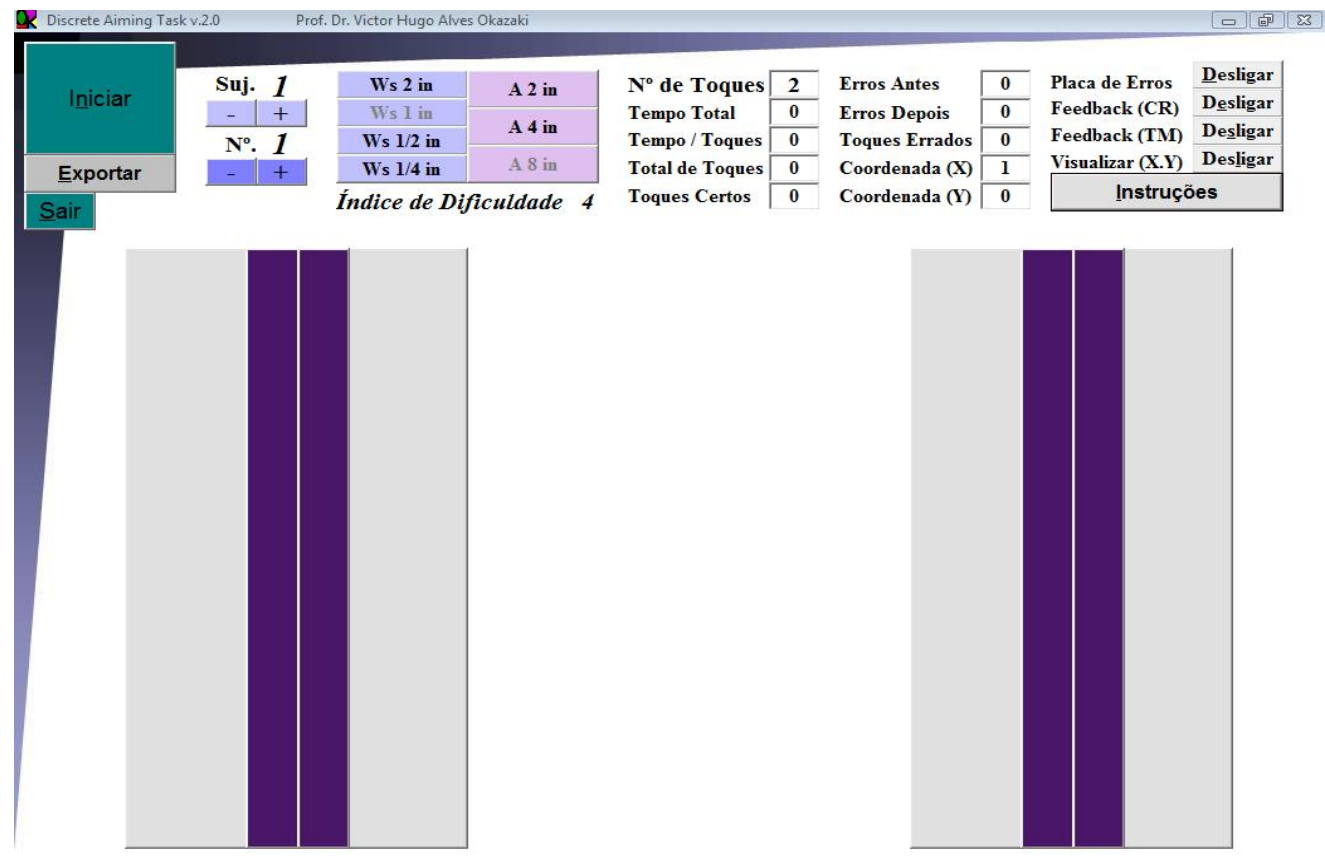

Figura 1. Representação da tarefa de Fitts realizada em ambiente virtual por meio do software Discrete Aiming Task (v.2.0)

tável em frente ao computador e realizaram três tentativas de familiarização praticando os movimentos específicos da tarefa nas condições experimentais. Os participantes foram instruídos a desempenharem os movimentos com maior velocidade e precisão possíveis. Após um sinal sonoro indicando que o movimento podia ser iniciado, os participantes realizaram a tarefa. O início do movimento foi determinado pelo acionamento do botão esquerdo do mouse sobre o primeiro alvo (lado esquerdo). O final do movimento foi determinado no acionamento do botão esquerdo do mouse no segundo alvo (lado direito). As tentativas foram descartadas quando: (a) o botão do mouse fosse acionado fora do segundo alvo, quando o acionamento do botão do mouse não funcionasse e quando houve erro na trajetória do mouse em ir e voltar sobre o segundo alvo. Praticamente, não ocorreram tentativas com erros durante a coleta de dados.

Foram realizadas 3 tentativas em cada condição experimental, manipulando-se o ID por meio dos três tamanhos de alvo e das três distâncias entre os alvos (total de 9 tentativas de teste). As sequências utilizadas de tamanho de alvo e de distância entre os alvos foram randomizadas entre os participantes através do quadrado de Williams adaptado. Em cada tentativa, os participantes tiveram o TM de cada toque como forma de feedback fornecido diretamente pelo software.

\section{Variáveis do Estudo}

As variáveis independentes do estudo foram o tipo de restrição espacial (tamanho do alvo x distância de movimento) e o índice de dificuldade $(I D=\log 2[2 D / A])$. Foram selecionadas as seguintes variáveis dependentes para a análise: (a) tempo de movimento (TM), (b) erro absoluto (calculado a partir de uma linha vertical central no alvo), (c) maior valor de velocidade, (d) tempo para o maior valor de velocidade (correspondente à fase de aceleração do movimento; cf. Teixeira, 2000), (e) tempo da maior velocidade até o final do movimento (correspondente à fase de desaceleração do movimento; cf. Teixeira, 2000), (f) razão entre o tempo da fase de aceleração e da fase de desaceleração, (g) tempo do submovimento primário (T1) e (h) tempo do submovimento secundário (T2). O submovimento foi definido 
pelo início do perfil da aceleração positivo, até o instante em que ocorre um valor de aceleração nulo, logo após um perfil de aceleração negativo (cf. Meyer et al., 1988). O tempo para o maior valor de velocidade (tempo para o pico de velocidade) também é utilizado para representar a fase de aceleração do movimento, enquanto o tempo restante corresponde à sua fase de desaceleração (Teixeira, 2000; Zelaznik, 1993).

\section{Tratamento dos Dados e Análise Estatística}

O software forneceu a posição linear do cursor do mouse em função do tempo com uma frequência de amostragem de $100 \mathrm{~Hz}$. Estes dados de posição foram filtrados com filtro recursivo do tipo Butterworth de quarta ordem com intensidade de $10 \mathrm{~Hz}$. Posteriormente, foram calculadas as derivadas de velocidade e de aceleração do movimento, e as variáveis dependentes desejadas foram extraídas. A associação $T M \times I D$ foi realizada por meio de uma Análise de Regressão Linear Simples. As variáveis dependentes foram comparadas por meio de uma ANOVA de Friedman, aplicada individualmente para cada grupo em função do ID (3, 4 e 5 bits). As comparações posteriores foram realizadas por meio do teste de Wilcoxon Pareado. O nível de significância das análises estatísticas foi estabelecido em $p<.05$.

\section{RESULTADOS}

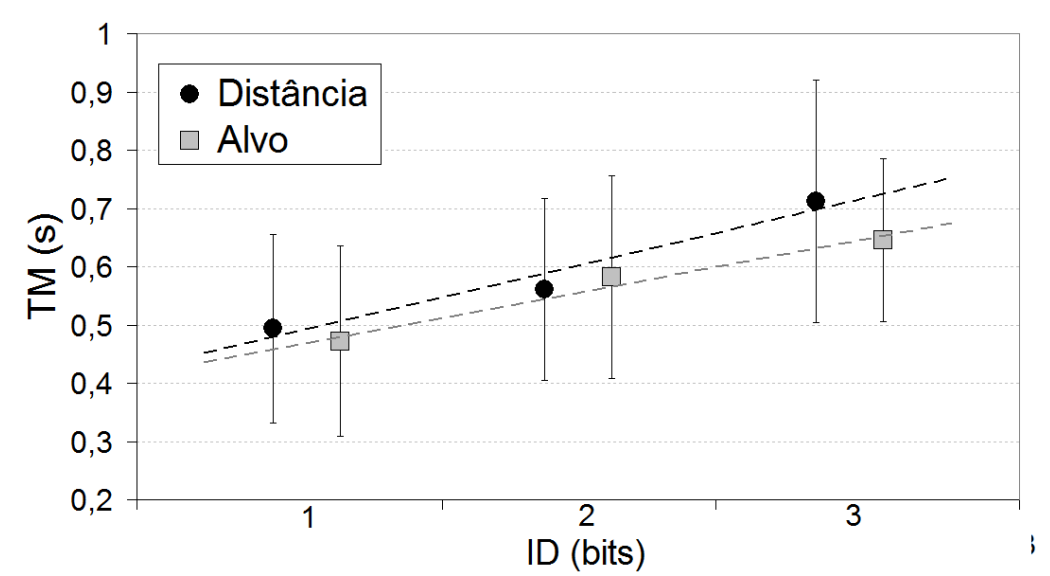

ID (bits)

\section{Relação TM x ID}

A análise de regressão linear para a relação TM x ID apresentou $r=990\left(R^{2}=.980\right)$ para a condição em que foi manipulada a distância $\left(F_{1,2}=48.2 ; p=.09\right)$ e $r=.998\left(R^{2}=.976\right)$ para a condição em que foi manipulado o alvo $\left(F_{1,2}\right.$ $=41.0 ; p=.10)$. Apesar dos grandes valores de associação, a análise de regressão linear não apresentou significância. Isto aconteceu, principalmente, em função do pequeno número de graus de liberdade (condições de $I D$ ) analisado. A Figura 2 apresenta a relação $T M \times I D$ nas condições em que foram manipulados os tamanhos dos alvos e as distâncias de movimento.

\section{Variáveis de TM, T1 e T2}

O teste de Anova de Friedman demonstrou efeito do ID para a condição de manipulação da distância $\left(X^{2}[N=20, g l=2]=17.8 ; p=\right.$ $.0001)$ e do alvo $\left(X^{2}[N=20, g l=2]=15.6 ; p=\right.$ .0004). No qual, a manipulação da distância demonstrou menor TM na condição de 5 bits, em comparação com às condições de 3 bits ( $Z$ $=3.7 ; p=.0002)$ e 4 bits $(Z=2.5 ; p=.014)$. A manipulação do alvo apresentou aumento no $T M$, com diferença entre todas as condições de ID $(Z>2.1 ; p<.034)$. O tempo do submovimento primário (T1) não teve efeito do $I D$ para a condição de manipulação na distância $\left(X^{2}[N=20, g l=2]=1.6 ; p=.448\right)$ e no alvo $\left(X^{2}[N=20, g l=2]=1.6 ; p=.441\right)$. Entretanto,

Figura 2. Relação entre o TM e o ID (média e desvio padrão) nas condições em que foram manipulados os tamanhos dos alvos e as distâncias entre os alvos na formulação do ID. 
foi verificado efeito do ID sobre o tempo do submovimento secundário (T2) para ambos, manipulação da distância $\left(X^{2}[N=20, g l=2]=\right.$ $12.4 ; p=.002)$ e do alvo $\left(X^{2}[N=20, g l=2]=\right.$ $14.7 ; p=.0006)$. De modo que, foi verificado maior TM, para a manipulação na distância, na condição de 5 bits comparada aos demais IDs $(Z>2.0 ; p<.035)$. E, menor TM na manipulação do alvo na condição de 3 bits, em comparação aos demais $I D s(Z>2.4 ; p<.014)$.

\section{Variáveis temporais da fase de aceleração e de desaceleração}

O ID demonstrou efeito sobre o tempo absoluto da fase de aceleração na condição em que foi manipulada a distância $\left(X^{2}[N=20, g l=2]\right.$ $=9.5 ; p=.009)$. No qual, houve maior tempo absoluto da fase de aceleração na condição de 5 bits, em comparação aos demais IDs $(Z>1.93$; $p<.05)$. No tempo de desaceleração absoluto, foi verificado efeito do $I D$ sobre a condição de manipulação da distância $\left(X^{2}[N=20, g l=2]=\right.$ $15.7 ; p=.0004)$ e do alvo $\left(X^{2}[N=20, g l=2]=\right.$ $9.0 ; p=.01)$. Em que foi verificado, para a manipulação da distância, maior tempo absoluto na fase de desaceleração na condição de 5 bits em relação aos demais IDs $(Z>2.0 ; p<.045)$; e, para a manipulação do alvo, menor tempo absoluto da fase de desaceleração na condição de 3 bits em comparação aos demais IDs $(Z>2.0$; $p<.045)$. A razão entre o tempo absoluto na fase de aceleração e na fase de desaceleração demonstrou um aumento na proporção da utilização da fase de desaceleração, para a condição manipulando o alvo $\left(X^{2}[N=20, g l=2]=12.7\right.$; $p=.002)$, no índice de dificuldade de 3 bits em comparação aos demais $(Z>2.4 ; p<.014)$. Ademais, também foi demonstrado maior coeficiente na razão entre as fases de aceleração e desaceleração para a condição de manipulação da distância, em comparação à manipulação do alvo $(Z>2.0 ; p<.045)$. A Figura 3 apresenta as variáveis temporais de $T M, T 1, T 2$.

\section{Variáveis de velocidade}

O ID demonstrou efeito no maior valor de velocidade sobre a manipulação da distância $\left(X^{2}[N=20, g l=2]=28.9 ; p<.0001\right)$. No qual foi verificado aumento na maior velocidade na medida em que o ID aumentou, com diferença significativa entre todas as condições $(Z>2.9 ; p<.0037)$. Também foi verificada diferença entre as condições manipuladas de distância e alvo, em que na condição de 3 bits foi verificada menor velocidade para a distância $(Z=3.3 ; p=.0008)$ e, na condição de 5 bits, foi verificada menor velocidade para o alvo $(Z$ $>2.4 ; p=.014)$. A manipulação da distância apresentou efeito do ID $\left(X^{2}[N=20, g l=2]\right.$ $=32.4 ; p<.0001)$, no qual o aumento no $I D$ levou ao acréscimo na velocidade media $(Z>$ 3.4; $p<.001)$. O ID também demonstrou efeito na manipulação do alvo sobre a velocidade média, no qual houve uma diminuição da velocidade na condição de 3 bits em comparação às demais $\left(X^{2}[N=20, g l=2]=10.3 ; p=.006\right)$. Também foi verificada menor velocidade média na condição de 3 bits $(Z=3.8$; $p<.001)$ e maior magnitude de velocidade na condição de 5 bits $(Z=3.8$; $p<.001)$, para a manipulação da distância em comparação à manipulação do alvo. A Figura 4 expressa os valores de velocidade (média e maior valor), em função do $I D$, para as condições de manipulação da distância e do alvo.

\section{Variável de erro/precisão}

O ID não demonstrou afetar a variável erro para a manipulação da distância $\left(X^{2}[N=20, g l\right.$ $=2]=1.3 ; p=.533)$. A manipulação do alvo demonstrou ser influenciado pelas condições de $I D\left(X^{2}[N=20, g l=2]=13.7 ; p=.001\right)$, nas quais foi verificado menor erro para a condição de 5 bits em comparação aos demais IDs ( $Z>$ $2.4 ; p<.014)$. Esta redução no erro na condição de 5 bits para a manipulação do alvo também foi significativa quando comparado ao maior valor de erro verificado na manipulação da distância $(Z=2.7 ; p=.006)$. A Figura 5 expressa o erro, 

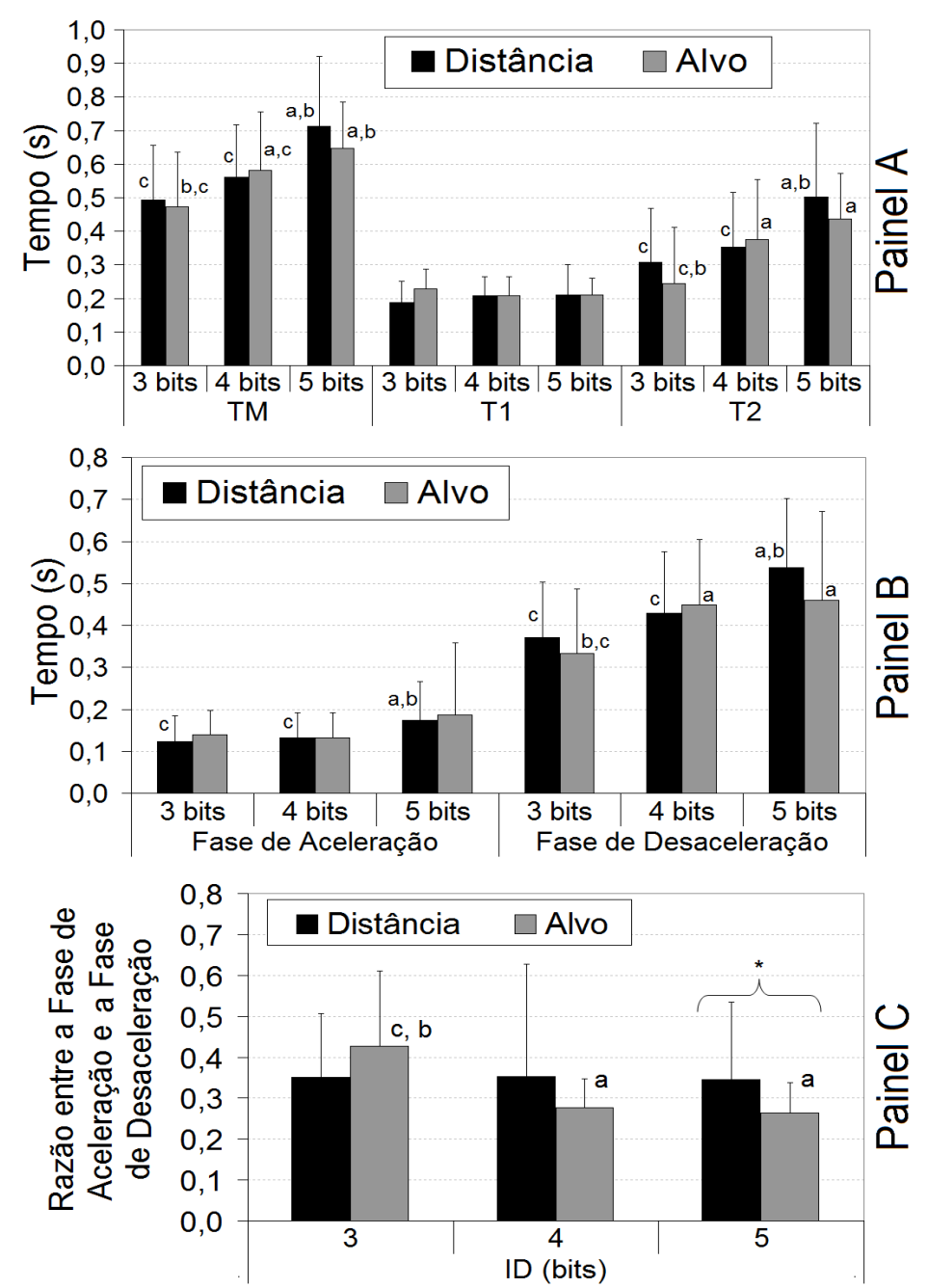

Figura 3. Variáveis temporais (média e desvio padrão) TM, T1, T2 (Painel A), fases de aceleração e de desaceleração (painel B), e a razão entre as fases de aceleração e desaceleração (painel C), nas condições em que foram manipulados os tamanhos dos alvos e as distâncias entre os alvos na formulação do ID. Diferença significante $(p<.05)$ comparado à condição com: ${ }^{\mathrm{a}} 3$ bits, ${ }^{\mathrm{b}} 4$ bits e ${ }^{\mathrm{c}} 5$ bits.

nas condições manipuladas de distância e alvo, em função do $I D$.

\section{DISCUSSÃO}

Foi verificada uma relação $T M \times$ x $I D$ com $\mathrm{R}^{2}>0,9$ para as condições em que foram manipuladas a distância de movimento e o tamanho do alvo (Figura 2). Entretanto, esta associação $T M \times I D$ apenas apresentou tendência para significância $(p=.10)$. Esta ausência de significância foi explicada pelo número reduzido de condições de ID (apenas 3, gerando apenas 2 graus de liberdade na análise de regressão linear) o que diminuiu o poder do teste estatístico utilizado. Apesar destes resultados, entretanto, a análise comparativa aponta para o efeito do ID sobre o TM.

O aumento do ID levou ao maior TM para as condições manipuladas de distância e de alvo (Figura 2). Este aumento no TM demonstrou ser realizado, à medida que houve acréscimo no ID, em função do aumento em T2 (fase dos submovimentos). Ou seja, a maior complexidade da tarefa (maiores restrições espaciais) demandou maior utilização do feedback sensorial para a regulação do movimento. Entretanto, não foram verificadas diferenças entre as magnitudes dos TMs nas diferentes condições de ID quando comparadas as situações de manipulação da distância e do alvo. Por conseguinte, independentemente da natureza da restrição espacial, com aumento na distância ou com 

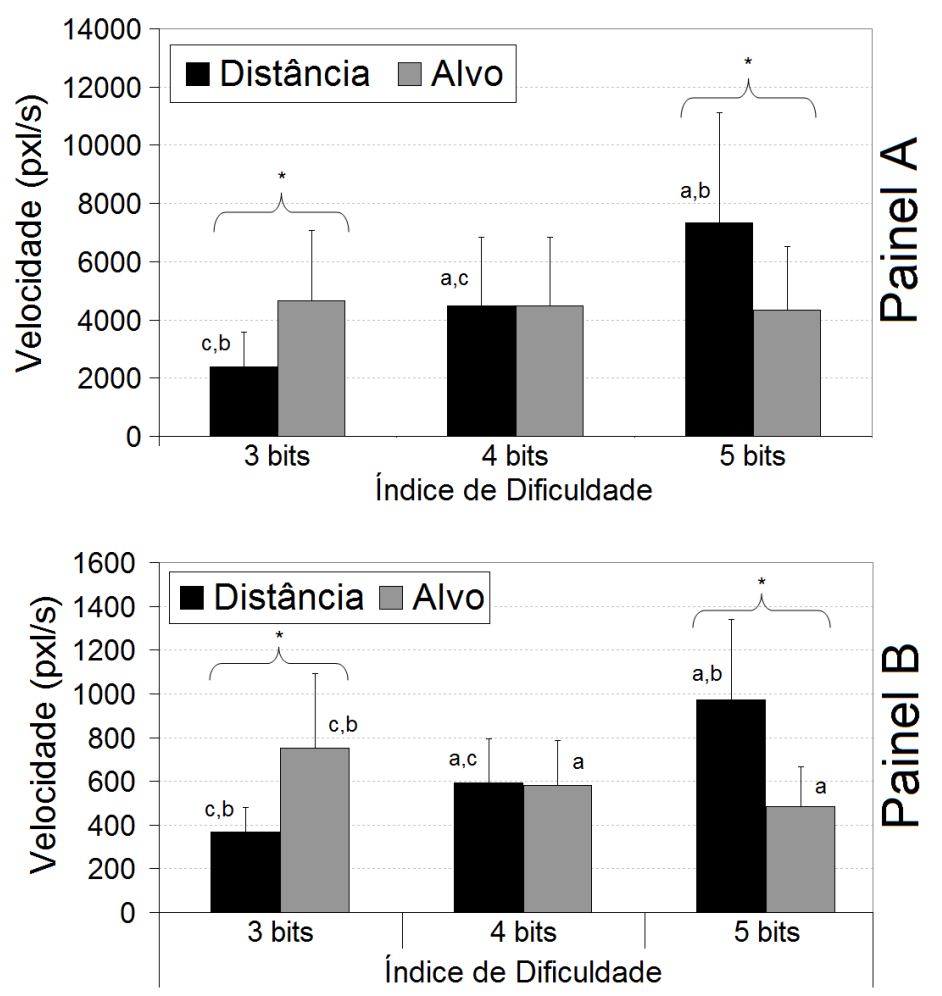

Figura 4. Valores (média e desvio padrão) da maior velocidade (painel A) e velocidade média (painel B), nas condições em que foram manipulados os tamanhos dos alvos e as distâncias entre os alvos na formulação do ID. Diferença significante $(p<.05)$ comparado à condição com: ${ }^{a} 3$ bits, ${ }^{b} 4$ bits e ${ }^{c} 5$ bits; *diferença entre as condições manipuladas de distância e de alvo.

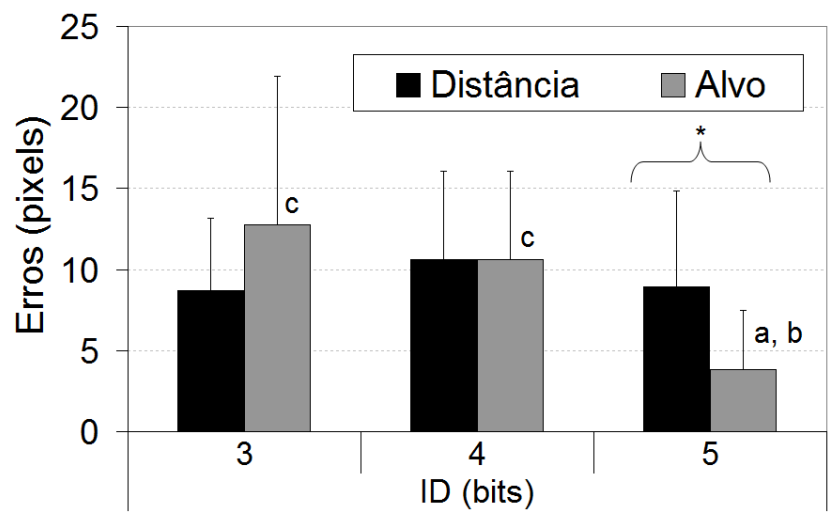

Figura 5. Erros (média e desvio padrão), nas condições em que foram manipulados os tamanhos dos alvos e as distâncias entre os alvos na formulação do ID. Diferença significante $(p<.05)$ comparado à condição com: ${ }^{2} 3$ bits, ${ }^{b} 4$ bits e ${ }^{c} 5$ bits; *diferença entre as condições manipuladas de distância e de alvo.

redução no tamanho do alvo, a fase pré-programada do movimento tende a se manter inalterada, ao passo que as alterações decorrentes na demanda da tarefa ocorreram na fase de controle via feedback. Estes resultados corroboram com proposições de modelos que explicaram a relação inversa velocidade-precisão por meio da utilização de feedback sensorial (Crossmon \& Goodeve, 1963/1983; Meyer et al., 1982; Okazaki et al., 2008; Woodworth, 1899).
Tem sido sugerido o aumento na fase de aceleração do movimento quando se objetiva a geração de velocidade no movimento (Teixeira, 2000; Zelaznik, 1993). Deste modo, seria esperada que maior fase de aceleração fosse verificada quando o ID fosse aumentado na condição de manipulação da distância de movimento. Os resultados do presente estudo demonstraram um aumento no tempo absoluto da fase de aceleração quando a distância de movimento 
foi aumentada. Entretanto, o acréscimo na distância de movimento também aumentou a fase de aceleração. Este aumento nas duas fases do movimento (aceleração e desaceleração), na condição de manipulação da distância, proporcionou a manutenção do tamanho relativo das fases, independentemente da condição de $I D$ (distância). Esta manutenção na proporção entre as fases de aceleração e de desaceleração pode ser verificada no painel $\mathrm{C}$ da Figura 3. Por outro lado, tem sido apontado que a maior demanda na precisão resulta no aumento da fase de desaceleração no movimento (Teixeira, 2000; Zelaznik, 1993). Os resultados do presente estudo demonstraram a manutenção no tamanho da fase de aceleração com o acréscimo na fase de desaceleração, em função do aumento no índice de dificuldade por meio da diminuição no tamanho do alvo (Painel B da Figura 3). Esta manipulação do instante em que o início das forças de desaceleração atua no movimento tem sido sugerida como estratégia que possibilita o controle da relação inversa velocidade-precisão (Zelaznik, 1993). O Painel $\mathrm{C}$ da Figura 3 também corrobora com a utilização desta estratégia de controle na precisão do movimento, no qual foi possível verificar uma diminuição no tamanho relativo da fase de aceleração e aumento relativo da fase de desaceleração. Por conseguinte, esta estratégia de deslizamento do início das forças de desaceleração pareceu ocorrer quando o ID foi aumentado por meio da diminuição no tamanho do alvo. Ao passo que, o maior ID aumentando a distância, demonstrou ser realizado com a mesma estratégia de manutenção na proporção entre as fases de aceleração e de desaceleração no movimento.

A diminuição no $T M$, na condição de restrição espacial em que foi manipulada a distância, foi realizada em função da maior trajetória a ser percorrida no movimento. Este aumento na trajetória de movimento também permitiu maior geração de velocidade (média e maior valor; Figura 3). Tem sido sugerido que o aumento na velocidade de movimento proporciona maior geração de força. Esta maior geração de força, por sua vez, proporcionaria a maior geração de ruídos neurais o que ocasiona em maior variabilidade na resposta (Meyer et al., 1982; Meyer et al., 1988; Schmidt, Zelaznik, \& Frank, 1978, Schmidt, Zelaznik, Hawkins, Frank, \& Quinn, 1979). Por conseguinte, foi levantada a hipótese de que as maiores velocidades de movimento resultariam em maior erro. Os resultados do presente estudo, todavia, não demonstraram modificação no erro (Figura 5), em função do acréscimo das maiores velocidades de movimento, realizadas por meio do aumento na distância. Deste modo, quando ocorre o aumento na distância, o movimento é desempenhado mais lento (maior TM) para tentar garantir sua precisão, porém, com maior incremento de velocidade (maiores velocidades média e magnitude). Tais resultados refutaram a hipótese estabelecendo maior erro em decorrência do aumento na velocidade de movimento.

O menor $T M$ na condição de manipulação no tamanho do alvo foi realizado com a manutenção na velocidade média e a redução no maior valor de velocidade. A manutenção na velocidade média foi explicada em função da distância de movimento ter sido constante entre as condições. Enquanto que, a diminuição no maior valor de velocidade foi sugerida como uma estratégia utilizada para aumentar a precisão no movimento. Tais resultados corroboram com as explicações de modelos baseados na geração de ruídos com o aumento na velocidade (Meyer et al., 1982; Schmidt et al., 1978; Schmidt et al., 1979). Por conseguinte, foi aceita a hipótese de que a diminuição no tamanho do alvo levaria às menores velocidades, resultando em maior precisão de movimento. A divergência entre os efeitos da velocidade sobre a precisão sugere que o aumento na restrição espacial por meio da manipulação na distância e no tamanho do alvo possui estratégias distintas de controle motor. 


\section{CONCLUSÕES}

A maior restrição espacial com maiores distâncias demonstrou aumentar o TM, por meio de maiores fases de aceleração, desaceleração e correções via feedback, mas sem alterar a proporção entre as fases de aceleração e desaceleração no movimento. As maiores distâncias também resultaram no aumento da velocidade (média e maior valor), entretanto sem alterar a precisão do movimento. Ao passo que, a maior restrição espacial, por meio da redução no tamanho do alvo, proporcionou maiores $T M$, fase de desaceleração e período para utilização do feedback, no qual foi fornecido maior tempo relativo para a fase de desaceleração no movimento. A diminuição no tamanho do alvo também reduziu o maior valor de velocidade, apesar da manutenção na velocidade média, resultando em maior precisão de movimento. Estes resultados sugerem que diferentes estratégias de controle são realizadas em função da restrição espacial manipulada pela distância e pelo tamanho do alvo. Foram sugeridos estudos que analisem o efeito das restrições espaciais de distância e de tamanho de alvo sobre tarefas bidimensionais, tridimensionais e habilidades motoras esportivas.

\section{Agradecimentos:}

Nada declarado.

\section{Conflito de Interesses:}

Nada declarado.

Financiamento:

Nada declarado.

\section{REFERÊNCIAS}

Boritz, J., Booth, K. S., \& Cowan, W. B. (1991). Fitts's law studies of directional mouse movement. Proceedings of Graphics Interface, 91, 216-223.

Bootsma, R. J., Fernandez, L., \& Mottet, D. (2004). Behind Fitts' law: kinematic patterns in goal-directed movements. International Journal of Human-Computer Studies, 61, 811-821. doi: 10.1016/j.ijhcs.2004.09.004

Buck, L. (1986). Target location effects in tapping tasks. Acta Psychologica, 62, 1-13.

Card, S. K., English, W. K., \& Burr, B. J. (1978). Evaluation of mouse, rate-controlled isometric joystick, step keys, and text keys for text selection on a CRT. Ergonomics, 21, 601-613.

Carlton, L. G. (1980). Movement control characteristics of aiming responses. Ergonomics, 23, 10191032. doi: 10.1080/00140138008924811

Corcos, D. M., Gottlieb, G. L., \& Agarwal, G. C. (1988). Accuracy constraints upon rapid elbow movements. Journal of Motor Behavior, 20, 255-272.

Crossman, E. R. F. W., \& Goodeve, J. (1963/1983). Feedback control of hand-movement and Fitts' law. Quarterly Journal of Experimental Psychology, 35A, 251-278.

Elliott, D., Helsen, W. F., \& Chua, R. A. (2001) Century later: Woodworth's (1899) two-component model of goal directed aiming. Psychological Bulletin, 127(3), 342-357. doi: 10.1037/00332909.127.3.342

Epps, B. W. (1986). Comparison of six cursor control devices based on Fitts' law models. Proceedings of the Human Factors Society 30th Annual Meeting, 327-331.

Fitts, P. (1954). The information capacity of the human motor system in controlling the amplitude of movement. Journal of Experimental Psychology, 47(6), 381-391.

Guiard, Y. (1997). Fitts' law in the discrete vs. cyclical paradigm. Human Movement Studies, 16, 97-131. doi:10.1016/S0167-9457(96)00045-0

Jagacinski, R. J., \& Mok, D. L. (1985). Fitts' law in two dimensions with hand and head movements. Journal of Motor Behavior, 17(1), 77-95.

Jagacinski, R. J., Repperger, D. W., Moran, M. S., Ward, S. L., \& Glass, B. (1980). Fitts' law and the microstructure of rapid discrete movements. Journal of Experimental Psychology: Human Perception and Performance, 6(2), 309-320.

Jax, S. A., Rosenbaum, D. A., \& Vaughan, J. (2007). Extending Fitts' law to manual obstacle avoidance. Experimental Brain Research, 180, 775-779. 
doi: 10.1007/s00221-007-0996-y

Johnsgard, T. (1994). Fitts' law with a virtual reality glove and a mouse: Effects of gain. Proceedings of the Graphics Interface Conference, 8-15.

Keele, S. W. (1973). Attention and Human Performance. Pacific Palisades: Goodyear Publishing.

Kerr, B. A., \& Langolf, G. D. (1977). Speed of aiming movements. Quarterly Journal of Experimental Psychology, 29, 475-81.

Kvalseth, T. O. (1975). A model of linear arm movements with preview constraints. Ergonomics, 18(5), 529-538.

Meyer, D. E., Smith, J. E. K., \& Wright, C. E. (1982). Models for the speed and accuracy of aimed movements. Psychological Review, 89(5), 449-492.

Meyer, D. E., Abrams, R. A., Kornblum, S., Wright, C. E., \& Smith, J. E. K. (1988). Optimality in human motor performance: Ideal control of rapid aimed movements. Psychological Review, 95(3), 340-370. doi: 10.1037/0033295X.95.3.340

Oldfield, R. C. (1971). The assessment and analysis of handedness: The Edinburgh inventory. Neuropsychologia, 9, 97-113.

doi: 10.1016/0028-3932(71)90067-4

Okazaki, V. H. A. (2006). Discrete Aiming Task (v.1.0) [Software]. Disponível através de http:// okazaki.webs.com/

Okazaki, V. H. A., Okazaki, F. H. A., Lima, E. S., Caminha, L. Q., \& Teixeira, L. A. (2008). Modelo estocástico de sub-movimentos otimizados em movimentos com restrição espacial simulados em computador. Revista Brasileira de Biomecânica, 9(16), 18-26.

Schmidt, R. A., Zelaznik, H. N., \& Frank, J. S. (1978). Sources of inaccuracy in rapid movement. In: G. E. Stelmach (Ed.), Information Processing in Motor Control and Learning (pp.183-203). New York, Academic Press.
Schmidt, R. A., Zelaznik, H., Hawkins, B., Frank, J. S., \& Quinn, J. T. (1979). Motor-output variability: A theory for the accuracy of rapid motor acts. Psychological Review, 86(5), 415-451. doi: 10.1037/0033-295X.86.5.415

Shannon, C. E. (1948). A mathematical theory of communication. Bell System Technical Journal, 27, 623-656.

Sheridan, M. R. (1979). A reappraisal of Fitts' law. Journal of Motor Behavior, 11, 179-188.

Sparrow, W. A., \& Sparrow, H. T. (1991). Trends in motor behavior research: A study based on citation analysis. Journal of Human Movement Studies, 21, 183-199.

Teixeira, L. A. (2000). Sobre a generalidade de estratégias de controle sensório motor. Revista Paulista de Educação Física, 3, 89-96.

Wade, M. G., Newell, K. M., \& Wallace, S. A. (1978). Decision time and movement time as a function of response complexity in retarded persons. American Journal of Mental Deficiency, 83(2), 135-144.

Welford, A. T., Norris, A. H., \& Shock, N. W. (1969). Speed and accuracy of movement and their changes with age. Acta Psychologica, 30, 3-15.

Wright, C. E., \& Meyer, D. E. (1983). Conditions for a linear speed-accuracy trade-off in aimed movements. Quarterly Journal of Experimental Psychology, 35A, 279-296. doi: 10.1080/14640748308402134

Woodworth, R. S. (1899). The accuracy of voluntary movement. Psychological Review (Monograph Supplement), 3, 1-119.

Zelaznik, H. N. (1993). Necessary and sufficient conditions for the production of linear speed-accuracy trade-offs in aimed hand movements. In: K. M. Newell \& D. M. Corcos (Eds.) Variability and Motor Control. Chicago-Ilinois: Human Kinetics Publishers.

(cc) EY-No Todo o conteúdo da revista Motricidade está licenciado sob a Creative Commons, exceto quando especificado em contrário e nos conteúdos retirados de outras fontes bibliográficas. 